\section{TECNOLOGÍA Y MARKETING: EL PAPEL DE LA RETÓRICA COMERCIAL EN EL DESARROLLO DE DISPOSITIVOS DE NAVEGACIÓN}

\author{
Pedro Mendonça \\ Instituto de Ciencias Sociales \\ Universidad de Lisboa
}

\begin{abstract}
The present article aims to link technology and marketing in the process of imagination and concretization of functions for mobile navigation devices. It focuses on the performance of marketing and sales, as rhetorical agents, in the technical path of building the product. Empirically, it is a case study of a Portuguese company, NDrive, whose main innovation is the introduction of photographic images in these devices replacing traditional maps. Since we identify in the field of innovation a platform of relation between the two spheres of inquiry (technology and marketing), it is in the theoretical studies of innovation that we produce much of the analysis of the different emerging questions, many connected to the central issues of contemporary technological transformation.
\end{abstract}

KEY WORDS: Technology; functionalities; marketing; rhetoric.

\section{INTRODUCCIÓN*}

En este estudio se pretende relacionar tecnología y marketing, teniendo en cuenta especialmente la interferencia del marketing en el desarrollo de funcionalidades (entendidas como utilidades o funciones de un dispositivo técnico) en sistemas de navegación (GPS). En esta relación no nos interesa tanto el marketing realizado para comercializar la tecnología, sino su intervención en la vertiente técnica en el momento mismo de la concepción de los productos.

Lo más importante para el estudio no es el marketing en sí mismo, sino las intenciones persuasivas cruzadas con las técnicas. Por lo tanto, estudiamos también prácticas de marketing que no sean realizadas por alquien que formalmente realice esa ocupación. Nos referimos a pragmáticas

\section{TECHNOLOGY AND MARKETING: THE ROLE OF COMMERCIAL RETHORIC IN THE DEVELOPMENT OF MOBILE NAVIGATION DEVICES}

RESUMEN: Este artículo pretende relacionar tecnología y marketing en los procesos de imaginación y desarrollo de funcionalidades en dispositivos de navegación móvil. Se centra en la actuación de los departamentos empresariañes de marketing, y de los trabajadores comerciales en general, como agentes retóricos que tienen un papel clave en los caminos técnicos de construcción del producto. En términos empíricos, se trata de un estudio de caso a una empresa portuguesa, Ndrive, que tiene como innovación principal la introducción de imagen fotográfica en los dispositivos de navegación, en vez de los tradicionales mapas. El campo de la innovación que se identifica es una plataforma donde se relacionan los dos ámbitos en cuestión (tecnología y marketing). Por ello, se utiliza la esfera teórica de los estudios de innovación para abordar el análisis de las diferentes problemáticas que van surgiendo en el desarrollo final del producto, muchas de ellas ligadas a las cuestiones centrales de la transformación tecnológica contemporánea.

PALABRAS CLAVE: Tecnología; funcionalidades; marketing; retórica.

que se enmarquen en el dominio persuasivo en lugar del técnico. Digamos que el marketing, dentro de la empresa, representa el ideal-tipo de persuasión más puro. En este caben los trabajadores comerciales en general, como por ejemplo los vendedores, que no se consideran como pertenecientes al departamento de marketing, pero que no están muy distantes de sus cometidos.

El proceso de indagación se ha centrado en cuatro objetivos: 1) comprender las intersecciones entre tecnología y marketing, principalmente las interferencias de los profesionales del marketing y los comerciales en los procesos de transformación e innovación de funcionalidades; 2) identificar el grado de participación de los consumidores; 3) verificar la existencia de campos de autonomía de la tecnología, frente a considerar que ésta es una mera 
construcción social, y 4) valorar el nivel de planificación que está presente en las intenciones técnicas y persuasivas que experimentan los actores involucrados.

Con estos elementos pretendemos comprender lo que puede inducir la persuasión comercial en la innovación tecnológica en un contexto determinado, y contribuir así a una mejor percepción de las configuraciones del desarrollo tecnológico actual. La secuencia del artículo es la siguiente. En el punto 2 se formulan algunas consideraciones sobre tecnología y marketing. En el punto 3 se expone el marco teórico empleado sobre los problemas analizados en los campos de la innovación y del desarrollo tecnológico. En el punto 4 se describe brevemente la metodología, incluyendo los conceptos operacionales y los procedimientos de observación. Seguidamente se realiza una breve presentación de la empresa y de sus productos. En el punto 6 se presenta el análisis de los resultados. El artículo finaliza con unas conclusiones referidas a los factores que intervienen en las innovaciones tecnológicas.

\section{Consideraciones Sobre tecnología y marketing}

Uno de los problemas principales en el estudio de la tecnología es que frecuentemente no se la coloca en perspectiva. Al contrario, muchas veces se la concibe como una extensión naturalizada de nuestras posibilidades o como una simple constante de las opciones de consumo que se nos presentan diariamente. En el estudio que presentamos no indagamos productos tecnológicos de especial trascendencia por su potencial de destrucción o que den lugar a arquitecturas tecnológicas de carácter "faraónico", como es habitual en algunos enfoques de la tecnociencia. Nos volcamos más bien sobre dispositivos de manipulación individual, asumiendo que pueden tener consecuencias globales, pero a partir del ámbito micro de lo cotidiano. Lo que nos interesa más especialmente no son sus efectos, aun cuando sin ellos estos artefactos tendrían poco interés. Lo que procuramos comprender son los procesos de concepción que les subyacen, teniendo en cuenta que en realidad éstos son su origen y la razón de su existencia. Saber lo que pasa en la producción y la relación con el consumo es determinar las líneas que tejen la transformación tecnológica. Con este propósito se considera relevante observar el marketing y los aspectos comerciales en general como hipotéticos espacios de mediación y persuasión. Ésta es la confluencia donde se sitúan los objetivos de este estudio.

El marketing, como disciplina relevante en una sociedad donde el consumo es un valor de carácter paradigmático, incorpora muchos aspectos de la ideología liberal (Lien, 1997). Se representa a sí mismo como puente entre productor y consumidor, ya sea para instigar al primero a seguir el segundo, o bien para elaborar impulsos en los consumidores que favorecen a los productores. A su vez, la tecnología no solamente constituye un elemento del sistema productivo, sino también se convierte en objeto producido para la comercialización.

Según los especialistas, el marketing es el "conjunto de los métodos y de los medios de que una organización dispone para promover, en el público que le interesa, los comportamientos favorables a la realización de sus propios objetivos" (Lendrevie et al., 1996: 28), lo que abarca las más diversas organizaciones, productos y públicos. En este estudio lo que nos interesa es el marketing estratégico y su influencia en la configuración del artefacto funcional, un tipo de marketing que pretende resultar en nuevos productos o productos mejorados, y no solamente en su comunicación o en aspectos organizativos (Tidd et al., 2003). A través de él, consideramos que las metas persuasivas pueden determinar la tecnología en su carácter más profundo, ejerciendo una autentica presión retórica sobre la invención, más allá del ámbito puramente comunicativo.

Llamamos retóricos a estos procesos porque, dentro de una empresa, son los departamentos de marketing y el comercial quienes desempeñan los roles más especializados en la persuasión. Ambos se movilizan en el sentido de inducir a los consumidores a comprar un determinado producto, utilizando para ello varios mecanismos. Por eso, son y utilizan retórica. A saber, la tarea de encontrar en cada caso los medios más adecuados para persuadir (Aristóteles, 1998, 1355b). Es, en cierto modo, una manera de llamar la atención del auditorio, en este caso formado por los consumidores.

En este punto conviene destacar que, a pesar de centrarnos en una retórica diseñada previamente, que tiene un carácter estratégico sobre lo funcional, pretendemos relacionarla con la fase posterior. Es decir, con la dimensión 
operacional del marketing, la que resulta, por ejemplo, en la comunicación institucional y en la publicidad.

A continuación nos centramos en el pensamiento sobre innovación tecnológica. Dado que procuramos establecer una relación entre tecnología y marketing en el desarrollo de nuevas funcionalidades, partimos del supuesto de que es en la creación y transformación de funcionalidades donde se puede descubrir la pauta de acción de diversos grupos sobre un objeto'.

\section{Producción, tecnología y consumo: ALGUNOS PROBLEMAS PROVENIENTES DEL CAMPO DE LA INNOVACIÓN}

Joseph Schumpeter (1996), economista clásico que desarrolla sus trabajos en este tema en la primera mitad del siglo $X X$, distingue entre invención e innovación. Entiende la invención como el acto creativo, en este caso de un objeto tecnológico. En contraste, la innovación es la asimilación social y el éxito económico del invento. Se podría asociar a la primera con el dominio técnico en su pureza, $y$, de manera similar, a la segunda con el dominio retórico. El historiador de la tecnología Bertrand Gille (1978) destaca cómo las empresas inventan para innovar, siendo esta separación cada vez menos real. De esta manera, se puede decir que los intentos retóricos se interponen a los técnicos, de ahí que en algunos autores la diferencia entre ambos no sea tan evidente.

Existen dos modelos principales utilizados para interpretar y eventualmente actuar en la promoción de la innovación. Por un lado, el clásico modelo lineal, representado por las propuestas de Vannevar Bush, que transcurre sin retroacciones, de forma unidireccional y por etapas. Éstas serían la investigación (ciencia y tecnología incitadas por la oferta - push-o por la demanda -demand-pull-), seguida por el desarrollo del producto $y$, finalmente, la producción y el marketing (Fagerberg et al., 2005). Por otro lado, más recientemente han surgido modelos interactivos y sistémicos, de los que los principales representantes son Kline y Rosenberg (1986) y su crítica a la propuesta de Bush, que prestan atención a un mayor número de agentes y consideran interacciones de tipo no lineal o reversible. Mientras que el primer modelo suele colocar al marketing y la comercialización en una posición que tiene escaso peso sobre la concepción y desarrollo de los productos, el segundo tiene en especial consideración la existencia de dicha intervención.

En los estudios sobre innovación son dominantes las visiones de carácter económico, vertiente que debe gran parte de su impulso al ya mencionado Schumpeter (1996) que, al romper con la visión que consideraba la innovación tecnológica como una variable exógena al desarrollo económico, la coloca en el centro como gran responsable de los ciclos económicos. El agente dinamizador sería el empresario, que no es necesariamente un gestor limitado a administrar, sino alguien que intuitiva e imprevisiblemente es creativo en sus decisiones. Schumpeter (1996), además, no cree que este empresario pueda perder su capacidad intuitiva frente a la gestión programada, una idea que en aquel tiempo expresó el miedo a la burocratización de la economía y de la automatización de los procesos.

Fruto de la tradición que subraya la automatización de los procesos es el enfoque del denominado determinismo tecnológico. Se trata de autores convencidos de esta automatización y de una cierta linealidad, que trabajan desde varias disciplinas, como la historia o la filosofía (ver Ellul, 1954). Esta visión sostiene que la tecnología se desarrolla con un grado de determinación tal que condiciona la sociedad, y que esta relación suele ser más fuerte que la existente en sentido contrario, al menos en algunos momentos claves de la historia. El historiador Robert Heilbroner (1967), en un artículo renombrado, sugiere esta tesis destacando tres argumentos: 1) el desarrollo tecnológico se hace por etapas que configuran un proceso relativamente independiente de los contextos sociales, aunque éstos adopten la tecnología de diferentes modos y ritmos -algo visible, por ejemplo, en la posibilidad de previsión de algún desarrollo tecnológico-; 2) el grado de conocimiento disponible constriñe el estado tecnológico, lo que indica una cierta secuencia y la existencia de posibilidades que van más allá de la decisión social; y 3) existen aspectos de la tecnología que provocan objetivamente ciertas coyunturas, principalmente en la división del trabajo y en el tipo de especialización industrial, como en el caso de las líneas de montaje y de las especialidades técnicas, posición próxima a la de Marx, cuando éste señala el papel del modo de producción en las relaciones sociales.

ARBOR Vol. 188753 enero-febrero [2012] 229-241 ISSN: 0210-1963 
Contrario a este determinismo, y como uno de los principales herederos de la línea de pensamiento de Schumpeter, cabe citar al economista Chris Freeman. Este autor atribuye mucha importancia a la innovación tecnológica en los fenómenos cíclicos de la economía. Pero, al mismo tiempo, sustituye los procesos de divulgación por procesos sociales concretos de la innovación y sumerge la figura del empresario en la de sistemas organizativos (Freeman \& Louçã, 2004). Contribuye así a la emergencia de la noción de "sistema nacional de innovación". En esta interpretación sitúa la observación en los esfuerzos para la innovación realizados dentro de los estados, donde se integran y coordinan diferentes entidades y estructuras organizativas, y por tanto no se otorga tanta importancia a "inevitabilidad técnica" frecuente en los autores de décadas anteriores (Freeman, 1987). En términos de modelo de innovación, esta perspectiva se desmarca del modelo lineal y opta por una visión más interactiva. Además, reconoce ámbitos aleatorios en el campo técnico, al mismo tiempo que apunta líneas definidas socialmente en la determinación de un trayecto tecnológico (Freeman \& Louçã, 2004).

Otro economista importante en este campo es Eric von Hippel $(1988,2006)$, autor que realza como pocos el papel del consumidor en los procesos de innovación tecnológica. Piensa que se trata de una posibilidad que debe de ser incentivada y para la que indica el concepto de "democratización de la innovación". Intenta refutar la idea de que la participación del consumidor se reduzca a los estudios de mercado realizados por departamentos de marketing. Al contrario, constata la existencia de consumidores que innovan tecnológicamente en el producto. En aquellos casos en que no existe otra forma de incorporar al consumidor, como en algunas tecnologías de punta, señala la importancia del llamado "usuario líder". Se trata de un consumidor más activo y atento que el "usuario tipo", que puede aconsejar transformaciones "necesarias" en el producto, lo que se puede interpretar como una forma de confrontar las dificultades que los consumidores revelan, así como la identificación de necesidades nuevas en productos tecnológicos (von Hippel, 2006). A pesar de la importancia atribuida al consumidor, también reconoce la existencia de otras fuentes de innovación, como el productor o el proveedor. En su entender, un consumidor, un productor o un proveedor es alguien que se beneficia del consumo, de la producción o del suministro respectivamente, lo que puede situar al productor en la posición simultánea de consumidor (von Hippel, 1988).

También en el lado de las visiones más complejas e interactivas de la innovación, en campo sociológico, surgen posiciones que contraponen a las fuerzas técnicas de carácter determinante otras fuerzas de carácter social. Son los denominados "constructivistas sociales de la tecnología", que aplican a este fenómeno el modelo de construcción social observado en las investigaciones sobre la elaboración del conocimiento científico. Los autores de esta corriente, como Pinch e Bijker (1984), destacan la idea de que la tecnología es un producto social. La innovación es, por lo tanto, moldeada por la sociedad, incluyéndose las influencias de los consumidores y del departamento de marketing. No obstante, el contraste en estos autores es que suelen excluir cualquier independencia de carácter técnico. Desde esta perceptiva, se interpreta que, en los procesos de construcción tecnológicos, varios grupos procuran reproducir en los objetos las soluciones para sus propios problemas, algo que rechaza admitir procesos tecnológicos autónomos (Punck \& Bijker, 1984).

Finalmente, cabe citar la llamada teoría del actor red, que reconoce en ocasiones determinaciones técnicas, aunque sin descartar el constructivismo social. Se trata de una línea sociológica interdisciplinar que integra elementos no humanos (la tecnología, por ejemplo) en las redes de acción entre humanos. Bruno Latour (1991), uno de sus principales representantes, pretende comprender las redes mediante el registro de las marcas o rastros dejados por cada actor (no-humanos inclusive), postura que pone a los humanos y los no-humanos a un nivel simétrico de influencia. Como consecuencia de la inclusión de un mayor número de factores, esta perspectiva admite más agentes en el modelo de análisis que utiliza. Junto con Akrich y Callon (2002a, 2002b), defiende que el pensamiento de Schumpeter se adapta menos a la realidad contemporánea cuando atribuye tanta relevancia al empresario. Como resultado, optan por un modelo aparentemente aún más difuso que el no-linear representado, por ejemplo, por Freeman. Al contrario, estos autores consideran simultáneamente la intervención del marketing, del consumidor, del empresario o del ciudadano lego, entre otros. De esta manera, sus estudios de caso se oponen a las visiones planeadas y estrictamente racionales del proceso de innovación. Negándose acentuadamente los modelos lineales, 
apuntan la ausencia de un impulso específico de la demanda o de la oferta, defendiendo la existencia de una complejidad del proceso, que se construiría por ensayo y error, donde domina la contingencia y la variedad de indicadores (Latour et al., 2002a, 2002b).

A partir de los autores expuestos, es posible identificar diferentes enfoques de acuerdo con los intereses heurísticos para este trabajo. En general, casi todos dan prioridad al polo de la producción. Aun así, von Hippel (2006) destaca el del consumo de modo distintivo. Por otro lado, existen posturas que consideran que la tecnología no existe como factor independiente, como la de los constructivistas sociales, y otras que piensan que la innovación tecnológica obedece también a procesos específicamente técnicos, como Heilbroner (1967). Otra postura procura combinar explícitamente los dos aspectos, como el caso de la teoría del actor-red. En relación al marketing, es importante señalar que gran parte de los autores no le otorga la debida atención como variable en la concepción del producto, además de en su divulgación, aunque existen algunos autores que lo señalan más claramente, como von Hippel (2006) o Latour, Akrich y Callon (2002a, 2002b), más el primero que los segundos². Finalmente, surge la cuestión de la planificación y previsión, más evidente en unos autores que en otros. Gran parte reconocen una importante presencia de un componente aleatorio e intuitivo. Algunos destacan elementos definidos en el campo técnico, principalmente Heilbroner (1967), mientras que otros lo hacen en el social, como Freeman y Louçã (2004). No obstante, la contingencia como regla representa a la opinión más corriente en esta materia. Estas ponderaciones parecen denotar un elemento no planificado dentro de la producción, concordando con Martins y Garcia (2006), cuando afirman la presencia de lo aleatorio en el centro de procesos especialmente planificados como los tecnológicos.

Resumiendo los problemas tratados, cabe resaltar cuatro aspectos relevantes para los objetivos de este trabajo: 1) el papel efectivo de los departamentos de marketing y comercial en la concepción de las funcionalidades de los productos; 2) el origen de la invención/innovación, que puede estar en la producción o en el consumo; 3) la autonomía de la tecnología en relación a otros factores, como el marketing; y 4) la existencia de procesos aleatorios e intuitivos en la producción persuasiva y técnica.
Estos puntos se observarán teniendo en cuenta la idea de intervención retórica. Para ello a continuación se expone la descripción de los conceptos operativos y de los procedimientos de observación, para seguir con una presentación de la empresa y de sus productos que sirven como estudio de caso estratégico para analizar el proceso expuesto hasta ahora.

\section{Metodología: conceptos operativos Y PROCEDIMIENTOS DE OBSERVACIÓN}

Observando las prácticas dentro de la empresa, uno de los aspectos que notamos es que existe una cierta imaginación o concepción creativa de las funcionalidades de los productos. En este contexto, por "imaginación" entendemos la proyección mental o el diseño creativo de una posibilidad tecnológica. Ésta puede ser diseñada o prometida: diseñada, si la consideramos cuando surge previamente a la finalización del desarrollo, sirviendo como horizonte de trabajo -por ejemplo, procurar añadir fotografía al sistema de navegación-; prometida, si la abordamos después de la finalización del desarrollo en forma de oferta al consumidor en la publicidad y en las instrucciones del producto -en el sentido de prometer que el sistema de navegación funcionará como lo esperado-. Los técnicos y los responsables del marketing, entre otros, pueden imaginar funcionalidades, pero también los consumidores, en este caso como posibilidades tecnológicas deseadas-por ejemplo, el deseo de la imagen fotográfica en la navegación-. Comprender el modo por el que estos procesos creativos se entrecruzan y cómo se finalizan nos permite comprender las actuaciones de los grupos sobre el desarrollo de funcionalidades de navegación.

Para una relación más específica a cada grupo en análisis, es útil articular también las nociones de "marco de referencia de funcionamiento" y "marco de referencia de uso" desarrolladas por Patrice Flichy (2002). Este autor, apoyado en la etnometodología y en el interaccionismo simbólico $^{3}$, defiende que, en el proceso de innovación, estos dos marcos están presentes como referencias. Los técnicos trabajan en un marco de referencia de funcionamiento, o sea, en un ámbito mental y práctico centrado en los problemas, en las teorías y en las referencias técnicas. En este marco se expresa la imaginación de funcionalidades que

ARBOR Vol. 188753 enero-febrero [2012] 229-241 ISSN: 0210-1963

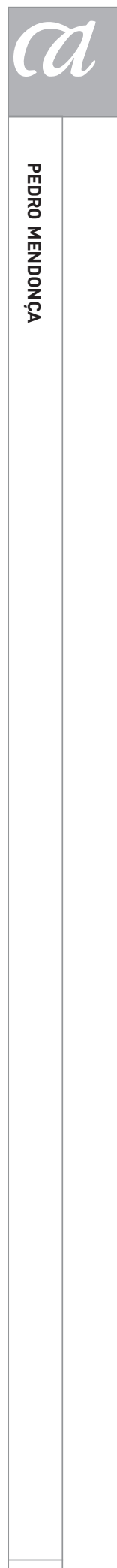

233 
se buscan desde un punto de vista técnico. A su vez, los utilizadores se relacionan con el objeto producido según las representaciones que tengan sobre su utilización, fundamentalmente los conocimientos necesarios para utilizar el dispositivo, además de las cuestiones prácticas a él asociadas. En este marco también se pueden imaginar las funcionalidades que se desean.

En algunos aspectos estos dos ámbitos se cruzan formando un marco de referencia común a partir de objetos fronterizos de comprensión mutua. Cuando los técnicos representan un usuario procuran integrar el grupo de referencia de éste para que se establezcan puntos de contacto. La funcionalidad, como elemento producido técnicamente y utilizado, se introduce de esta manera. En el caso contrario, el usuario no sabría cómo emplearla. Algunos de estos cruces se forman a partir de foros donde surge comunicación entre funcionamiento y uso, tales como el apoyo técnico, los estudios de mercado y los foros en internet.

A estos dos conceptos de Flichy pretendemos añadir el de "marco de referencia retórico" referido a los profesionales de marketing y comerciales en general. En el análisis empírico notamos que éstos tratan con conocimientos, problemas, modelos y un tipo de imaginación dirigidos a persuadir. Son elementos no reductibles al marco de referencia de funcionamiento. Se cruzan con éste y con el uso en marcos de referencia comunes. Por ejemplo, la conciencia que algunos departamentos que trabajan en aspectos retóricos tienen de los problemas técnicos, la percepción que los técnicos adquieren de la necesidad de vender o las representaciones del consumidor que ambos producen. En el caso de que el grupo retórico imagine funcionalidades a desarrollar (a perseguir de manera expresa), actuando en la concepción técnica del producto y no solamente en la promesa comunicativa al consumidor, será visible una influencia del retórico sobre el técnico al nivel del artefacto funcional.

Con este enfoque conceptual, se realizó un estudio de caso a una empresa portuguesa, Ndrive, especializada en sistemas de navegación. Su innovación más relevante es la aplicación de "imagen real" (fotografía) con perspectiva oblicua a dispositivos personales de navegación. En un análisis intensivo, se realizaron entrevistas a los actores involucrados en la concepción y el desarrollo del producto, algunas observaciones in situ, interpretaciones de docu- mentos internos, así como de noticias. Adicionalmente, se realizó una observación del funcionamiento material de los artefactos. La diversidad de técnicas ha permitido un proceso de triangulación.

A continuación se realiza una presentación de la empresa y sus productos, para después mostrar algunos de los resultados del análisis de acuerdo con los conceptos expuestos.

\section{LA EMPRESA Y SUS PRODUCTOS}

La empresa Ndrive ${ }^{4}$ fue fundada en 2005 a partir de otra, InfoPortugal ${ }^{5}$, nacida en 2001. La primera es una empresa de tamaño medio ${ }^{6}$, pero de momento se encuentra en plena expansión. Con sede en la ciudad de Oporto, se puede considerar la única en el mercado portugués dado que ninguna otra empresa produce sistemas de navegación. En cierto modo es la típica empresa media de tecnologías de información y comunicación: tiene una capacidad de organización flexible, pero una menor capacidad de financiación para los sistemas técnicos extensos, al contrario que lo que suele ocurrir con las empresas grandes (Tidd et al., 2003).

InfoPortugal, a su vez, se constituyó a partir de los mismos fundadores de Ndrive y está especializada en el componente cartográfico. En el fondo, la distinción entre estas dos empresas es puramente artificial. Hay que realzar el hecho de que InfoPortugal, al igual que Ndrive, fue creada por un profesor universitario y consultor en marketing?. Este profesor respondió al encargo de un estudio de "oportunidad" por parte de otra empresa de software sobre las necesidades del mercado portugués en el dominio de los móviles de tercera generación. Las conclusiones indicaron la existencia efectiva de un mercado en el ámbito de los mapas digitales. Dado que la empresa que solicitó el estudio desistió en desarrollar la oportunidad de negocio, este profesor decidió hacerlo de manera independiente. InfoPortugal nació, por tanto, a partir de un conocimiento en cierto modo producido por el marketing. Ndrive surgió, posteriormente, de la expansión del negocio de los mapas para el software de navegación, lo que resultó igualmente de prestar atención a las oportunidades de mercado desde el interior de InfoPortugal. 
Al principio, cuando Ndrive era muy pequeña, la función de marketing operaba apenas en los conocimientos del promotor, compartiendo ese "cuerpo" con otras funciones organizativas como las de gestión. Con el crecimiento de la empresa el número de trabajadores aumentó, al igual que la estabilización de tareas. Actualmente ya existe un departamento formal de marketing, aunque estas funciones laborales siempre han estado presentes en la empresa.

El nombre de la empresa comenzó a destacarse en los periódicos cuando, en 2006, el Presidente de la República Portuguesa Cavaco Silva ofreció al Rey Juan Carlos un producto Ndrive como ejemplo de tecnología portuguesa en una visita a España. En 2009, la empresa ganó una mención de honor de la Asociación Empresarial para la Innovación (COTEC) por uno de sus productos.

Esta empresa también ha recibido desde su creación algunas ayudas financieras estatales a modo de incentivos para la formación de empresas innovadoras y su internacionalización. Dichas iniciativas facilitan que la empresa esté incorporada a la estratégica nacional para la innovación y acciones emprendedoras, por la que las autoridades públicas pretenden implantar (y en cierta manera "inventar") un "sistema nacional de innovación" portugués (ver Rodrigues et al., 2003).

En lo referido a los productos de sistemas de navegación, Ndrive tiene como innovación principal la imagen fotográfica con visualización oblicua aplicada a la navegación. No obstante, este producto no tuvo el éxito comercial esperado. La "imagen real" surgió como consecuencia de una propuesta de otra empresa, $\mathrm{Blom}^{8}$, que detenta en Europa la patente de las imágenes con las características que incorporan estos dispositivos, aunque sin la aplicación a la geo-navegación desarrollada por Ndrive. Existen, sin embargo, otras características de sus productos que merecen alguna referencia. En términos de hardware, es de resaltar la introducción de un alcoholímetro en uno de los productos de navegación; la concepción de móviles (éste no es un producto innovador, pero es importante debido a que supone un proceso de diferenciación único en Portugal); y la creación de un dispositivo de navegación más reducido que los disponibles en competencia (Tomtom ${ }^{9}$, Garmin ${ }^{10}$, etc.). Con respecto al software de navegación, se pueden destacar las posibilidades de acceder a menús más rápidos y simples; explorar una mayor personalización del producto; importar datos de Google; acceder a puntos de interés categorizados con búsquedas consideradas más eficaces; seguir caminos del modo llamado "de aventura" (destinos que se siguen sin camino predeterminado); 0 introducir información de carácter comunitario respecto a puntos de referencia para grupos con intereses comunes (por ejemplo, los pescadores pueden tener acceso a lugares considerados buenos para pescar por sus compañeros). Recientemente, la empresa dejó el hardware y apostó sólo por el software y su compatibilidad con varias plataformas, como el Iphone y varios tipos de móviles.

\section{Resultados}

A continuación se muestran los resultados que el análisis de estos aspectos arroja. El punto se ha dividido teniendo en cuenta los cuatro objetivos/problemas planteados anteriormente, utilizando en el análisis los conceptos operativos y los procedimientos de observación descritos.

En relación al primer problema principal de este estudio, referido a la intervención de los responsables retóricos en el desarrollo de nuevas funcionalidades, se ha podido verificar que la innovación más radical de la empresa desde el punto de vista técnico (aunque puede considerarse como incremental en el contexto de este dispositivo -la aplicación de la fotografía con visión oblicua al sistema de navegación-), no fue resultado del proceso de concepción creativa o imaginación de funcionalidades por parte de los técnicos o de los retóricos de la empresa. La funcionalidad tecnológica efectivamente ha sido desarrollada en Ndrive, aunque el proceso de imaginación se produjo en la empresa Blom, que fue la que lanzó el desafío técnico. Lo que ocurrió en Ndrive es que se aceptó una imaginación exterior y se trabajó técnicamente para desarrollarla. Aún más, cuando se ha consultado a la empresa Blom, se verifica que la imaginación de esta funcionalidad fue fruto de la necesidad de aplicar la imagen real con visión oblicua a más productos como una manera de maximizar las ventas. Se trata por tanto de una iniciativa marcada por un marco retórico típico del marketing de oferta.

La empresa Ndrive tuvo el mismo tipo de iniciativa en algunas de sus opciones: cuando decidió apostar por los móviles, tanto fabricándolos como realizando el sistema

ARBOR Vol. 188753 enero-febrero [2012] 229-241 ISSN: 0210-1963 
de navegación compatible con teléfonos móviles, o cuando incorporó el alcoholímetro al dispositivo, lo que pretendió fue asociar el producto a una plataforma que ampliase el espectro de uso. Esto fue también un intento de vender más, lo que se enmarca en la misma dinámica que motivó a Blom. Se podría llamar por tanto retórica de oferta de funcionalidad, algo que, a pesar de partir de un estímulo externo, forma parte efectiva del avance técnico.

En Ndrive, el grupo retórico ha producido otras funcionalidades: el hardware más reducido, el modo de navegación "de aventura" y las informaciones de carácter comunitario surgieron de su iniciativa. Los técnicos, a su vez, también imaginaron y propusieron funcionalidades: por ejemplo, algunas opciones de interface o aspectos técnicos que convirtieron el producto en un dispositivo más rápido. En general, asistimos a procesos de creación de problemas compartidos entre retóricos y técnicos: algunas cosas concebidas por los primeros son limitadas por los segundos, mientras algunas cosas imaginadas técnicamente son a su vez limitadas en el ámbito retórico, aunque a veces se solapan en marcos de referencia comunes. Estas interacciones surgen en brainstormings o mediante un "individuo bisagra" que dispone de conocimientos duales. En este caso se trata del principal responsable en el pasado de la gestión del producto, persona que ha incorporado, literalmente, un marco de referencia común a los técnicos y a los retóricos.

En las funcionalidades estudiadas emerge una pauta interesante: los retóricos proponen funcionalidades más visibles y aparentes, que a veces son incompatibles técnicamente. Por su parte, los técnicos están más próximos a aquello que no se percibe, pero que es esencial para que las funciones operen adecuadamente.

El papel del marketing en el ámbito de la comunicación también parece actuar en el dominio funcional de una manera destacada, pero es principalmente a posteriori. Es decir, aprovecha funcionalidades para producir comunicación y no a la inversa ${ }^{11}$. Por ejemplo, la decisión de crear móviles e introducirles GPS surgió de la necesidad de asociar el GPS a un dispositivo de uso más frecuente y de tener una gama más amplia. No obstante, el imperativo de la comunicación -"el primer móvil portugués"- fue posterior en lo referido a la intención de llevarlo a cabo. Aquí vemos un cierto nacionalismo tecnológico comunicacional dependiente del ámbito funcional. También está relacionado con el papel del Estado, lo que se refleja en el regalo del Presidente de la República al Rey de España. Así pues, al igual que la empresa se proyecta en un discurso de valor nacionalista del producto, vemos cómo el Estado también se apropia de él. Existe una cierta inmersión comunicativa en un "sistema nacional de innovación", que además va acompañada de ayuda financiera como parte de una estrategia nacional dirigida por el Estado.

Otro aspecto que consideramos importante para articular el ámbito funcional y el comunicativo se puede llamar como "factor uau!", utilizando una de las expresiones de los responsables retóricos. Esta expresión onomatopéyica denota la reacción de asombro a una funcionalidad, que no tiene por qué surgir del consumo o tener mucho éxito comercial, pero que sí muestra el poder de la empresa para producir funcionalidades. Esto apunta principalmente a otras empresas clientes. En cierto modo, es lo que sucede con la imagen real en el GPS: sirve de identificación de la empresa, pero no es un éxito comercial. En este registro, se percibe una tendencia de los retóricos para dramatizar el carácter innovador de algunas funcionalidades, que sin embargo se consideran como imitaciones por parte de los técnicos. Se trata de cierto factor "uau!" vacío de contenido técnico, en que las estrategias de comunicación se desvinculan de la ingeniería.

En cuanto al segundo problema, el que indaga si el origen de los productos está en la producción o el consumo, es posible afirmar que predomina la producción como fuente de innovación y transformación. Los marketeers y los comerciales, supuestos responsables de la participación del consumidor y de la generación de una retórica de demanda, confiesan la imposibilidad de realizar estudios de mercado con el tiempo y la profundidad necesarios debido a los cambios tan rápidos que se producen en este campo, aunque suelen consultar estudios externos. La relación directa con el consumidor ocurre predominantemente después de la comercialización del producto, y se debe sobre todo a problemas en el funcionamiento (prometido) de los productos ${ }^{12}$.

Los procesos y los espacios que ayudan a la elaboración de marcos comunes de entendimiento entre producción y consumo son los estudios de mercado externos ya referidos, que son escasos, y además están centrados en 
detectar tendencias de ventas y, en el mejor de los casos, en preferencias de elección múltiple. Es decir, no resultan de un proceso endógeno, sino de las opciones que se crean de modo exógeno (ver Horkheimer \& Adorno, 2002 [1944]). Cabe citar otros ejemplos: el apoyo técnico, que por su naturaleza trabaja en la resolución de problemas después del lanzamiento del producto y dentro de las funcionalidades prometidas. Internet, principalmente foros de discusión entre usuarios líderes, donde a menudo intervienen responsables de la empresa, aunque en diálogos que se centran en la misma lógica para resolver los problemas de funcionalidades prometidas ${ }^{13}$ (por ejemplo, la aplicación del producto al Iphone hizo crecer este dominio a escala internacional). El departamento que realiza los test, articulado con el apoyo técnico, que intenta aprender de los errores de los productos anteriores, integrando su previsión en los test que realiza a los nuevos dispositivos. Los familiares y conocidos de algunos de los profesionales, solicitados para experimentar los productos. Y, finalmente, los propios técnicos y retóricos, asumiendo a veces el papel de usuarios, tal como von Hippel (1988) indica.

Otra serie de actores relevantes en la construcción del producto distintos a los consumidores finales son otras empresas, que a veces piden como condición de la compra de grandes cantidades que se introduzcan ciertas funcionalidades. Éstas son frecuentemente mal vistas por parte de los técnicos, dado que las consideran poco coherentes con el sistema de navegación. Esto es un ejemplo de cómo el producto se desarrolla de manera centrada en la empresa, o bien a partir de una relación abierta con otras empresas (como es el caso de Blom), y no tanto en el consumidor.

Los datos que presentamos demuestran que no todas las relaciones con el consumo son llevadas a cabo por los departamentos de marketing y comerciales, como es el caso de las realizadas por el apoyo técnico o por el departamento de test, aunque sea en el dominio retórico donde predominan como proceso formalizado. Parece indiscutible que cualquier técnico tiene en mente al usuario.

Tanto retóricos como técnicos representan en sus mentes el consumidor como un "consumidor normal", en sus palabras, "el hombre medio que no entiende mucho de tecnología, aunque sí lo suficiente para comprarla y uti- lizarla". Es decir, el "usuario tipo" indicado por von Hippel (2006). El marketing, por tanto, procura influenciar el departamento técnico con dos exigencias principales, independientemente de quién imagine las funcionalidades: la de "simplicidad" y la de "utilización intuitiva" (algo que muchas veces los técnicos dicen que les es natural). Se trata de crear un objeto sin la complejidad que corresponde a un pensamiento excesivamente técnico, y que además tenga un interface que permita cierta continuidad orgánica entre las funcionalidades y el usuario. La continuidad ocurre, por un lado, al nivel de la percepción de los símbolos paradigmáticos $y$, por otro, de los gestos considerados "naturales". Estos últimos están determinados por los códigos gestuales estabilizados socialmente. Como ejemplos relevantes cabe resaltar los siguientes: los símbolos paradigmáticos serian los símbolos en el menú, que tienen que ser reconocibles; los códigos gestuales serían el tamaño de las letras en la pantalla táctil, que deben ser lo suficientemente grandes para que con un dedo se pueda teclear) ${ }^{14}$.

Los marketeers y los comerciales cuanto adoptan el papel de usuarios representan y vigilan una supuesta "corporalidad universal", al observar los comportamientos individuales y los discursos, de manera que puedan producir y detectar la imaginación de nuevas funcionalidades. El objetivo es crear un objeto lo suficientemente com-

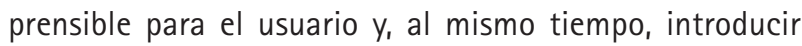
nuevas configuraciones que soportan diferentes formas de utilización. De todos modos, se trata de procesos de inducción débil, es decir, que inducen desde un punto de vista particular y reducido.

En relación al tercer problema -el de la autonomía del ethos técnico- es posible afirmar que existe, aunque de manera atenuada. Podemos dividir este ámbito en dos niveles: el de los técnicos, como individuos dotados de intenciones, y el de la técnica como trayectoria de oportunidad y condicionante material. A la actuación de los técnicos, de acuerdo con el antropólogo Alfred Gell, se la llama agencia primaria. A la actuación de la técnica como trayectoria de oportunidad se la llama agencia secundaria, dado que refleja la agencia primaria sin auto-suficiencia (Grell, 1998). En lo referido al condicionante material, es posible remitirlo al nivel del acontecimiento, debido a que resulta de las leyes propias de la materia y no tanto de la agencia social.

ARBOR Vol. 188753 enero-febrero [2012] 229-241 ISSN: 0210-1963 (1) 
En el primer nivel encontramos algunos casos donde los técnicos imponen sus propuestas. A veces imaginan funcionalidades que son tanto diseñadas como prometidas, como ya se ha mencionado, lo que los convierta en algo más que simples ejecutores de imaginaciones ajenas. Además, sus conocimientos técnicos exclusivos les permiten acceder a una imaginación propensa a nuevas posibilidades difíciles de descubrir en el exterior no técnico, lo que contribuye a que se cree un espacio de creatividad y autonomía inmune a jerarquias profesionales.

En el segundo nivel, si miramos a la innovación de la imagen real, junto a la retórica de expansión funcional ya referida, vemos como también se relaciona con una oportunidad técnica de cruce de trayectorias diferentes, aunque no son antagónicas - por ejemplo, entre la fotografía aérea con visión oblicua de Blom y el sistema de navegación de Ndrive-. Se trata de una posibilidad que quizá se pueda imaginar por un especialista. Es pertinente considerar las tendencias contemporáneas dirigidas a obtener un mayor realismo de la imagen (fotografía) y para incrementar el control y anticipación (GPS) como referencias presentes en esa decisión. Algo próximo de la posición de Heilbroner (1967) cuando se refiere a la previsibilidad del desarrollo tecnológico como argumento a favor del determinismo.

En este caso existió anticipación humana para actuar sobre una posibilidad técnica. Aunque el proceso haya sido dialéctico, y la decisión humana haya sido preponderante, vemos formarse una línea tecnológica exterior -como agencia secundaria (fotografía y GPS)- que puede alimentar la tesis de un cierto determinismo al que podemos llamar "profético auto-realizado"15. Es decir, entre varias posibilidades técnicas se escoge una, que es la que sobresale, por lo que hay una cierta determinación de la decisión que se va a materializar. En este aspecto se observa una interacción con el primer nivel, o sea, con las expectativas de los técnicos, pero sobre todo con los fines retóricos que tienen su origen en Blom y afectan a Ndrive.

Por otro lado, de manera más detallada, vemos respuestas y determinaciones de la materia técnica en el desarrollo del trabajo técnico al nivel de las funcionalidades diseñadas, donde acontecimiento y agencia secundaria se entrelazan: existen imaginaciones de funcionalidades que se intentan desarrollar y que en la práctica son imposibles, por ejemplo, dar a la imagen real una dinámica cinematográfica, como se llegó a proponer en Blom. Hay una resistencia de la propia estructura técnica a lo largo del desarrollo en tensión con lo imaginado, como sucede con la dificultad de entrecruzar la complejidad de los mapas con la del software y su base de datos. Los propios retóricos también tienen consciencia de los límites técnicos (por ejemplo, en lo referido a la capacidad de memoria y rendimiento del producto). Estos aspectos demuestran que la libertad de los técnicos no es total en relación a la materia técnica, lo que ayuda a contrariar la tesis del constructivismo social de la tecnología al distinguirse diferentes tipos asimétricos de agencia.

Finalmente, en relación al último problema -la existencia o no de procesos de planificación técnica y retórica- es posible afirmar que, a pesar de existir un deseo de previsión y control, los que intervienen describen un ambiente de gran incertidumbre. Es algo alimentado por la escasez de tiempo y por la complejidad del ambiente, lo que dificulta las deliberaciones profundas. Para los técnicos, el marketing y los comerciales exigen poco tiempo. Para éstos, los técnicos necesitan demasiado tiempo. Ante esto, vemos un esfuerzo de planificación que en los primeros años es bastante informal. Además, los planes sufren desvíos frecuentes y en la mayoria de los casos se basan en objetivos intermedios (en relación a los objetivos finales de funcionamiento, de venta y lucro) y por tanto no definen las tareas que son necesarias. Éstas son introducidas mayoritariamente por ensayo y error, aunque no dejan márgenes para muchos fallos sucesivos. La apertura a propuestas externas también tiende a producir fragilidad en la planificación. Se trata de aspectos en los que la perspectiva y el deseo de Shumpeter (1996) salen reforzados, pues muchos de los procesos no son programados. Desde este punto de vista, la determinación profética autorealizada consiste en una apuesta incierta y no planificada, teniendo en cuenta que las posibilidades son varias y que la opción que se escoge debe ser aceptada en el mercado.

Con el crecimiento de la empresa estas dinámicas tienden a estabilizarse en lo que Thévenot (2006) denomina de "régimen normal", es decir, estandarizado: los medios resultan de la experiencia y se establecen como regla después de superar el criterio del éxito. Esto se confirma en la adopción de un programa de computador (Scrum) que impone un modelo de acción a los técnicos de la empresa en el desarrollo de funcionalidades. Por lo tanto, se observa que el crecimiento ha llevado a más automatismos de tipo técnico y retórico. 


\section{Observaciones finales}

Considerando los elementos analizados, podemos decir que el grupo retórico actúa en diálogo con el de funcionamiento. En general, asistimos a una dialéctica de problemas entre los dos dominios. En la demarcación de la demanda de acuerdo con el consumidor y de manera distinguible a la competencia, tiene predominio el retórico. Sin embargo, crece el técnico en lo referido a la imaginación de funcionalidades invisibles al usuario, pero fundamentales para un buen funcionamiento del producto.

En este movimiento de balance entre los dos grupos, si miramos las decisiones más importantes, lo retórico acaba por ser más fuerte, principalmente debido a que predomina una mayor atención hacia el exterior ${ }^{16}$. Fue en este ámbito y en sus lógicas persuasivas en el que nacieron InfoPortugal y Ndrive. Por lo tanto, lo que observamos es que, desde el inicio, un proceso retórico especializado ha marcado el desarrollo de funcionalidades de navegación. Desde este punto de vista, podemos hablar de una retórica de la funcionalidad que no se restringe al dominio de la comunicación (Mendonça, 2010).

Hay un predominio de procesos retóricos de oferta donde imperan las funcionalidades diseñadas y prometidas al margen del deseo previo manifestado por el consumidor. El marketing y los comerciales, y también algunos técnicos, se movilizan en un propósito retórico que forja prácticas mediante observación y experimentación de símbolos y códigos gestuales. Una articulación entre producción y consumo que se hace sobre características tecnológicas determinadas por la empresa, lo que favorece el acelera- miento tecnológico sobrepuesto al cultural indicado por Stiegler (1994).

Debemos tener en cuenta que este desarrollo tecnológico tiene aspectos técnicos que condicionan la actuación humana. Podemos hablar de una materia técnica que establece algunos acontecimientos, lo que a veces aparece como aspecto determinable por decisiones de carácter "profético". Esto está influenciado por un ambiente donde predomina la urgencia por diferenciarse, donde los que intervienen se ven obligados a innovar por innovar. La especialidad retórica del marketing y de los comerciales produce un tipo de estrategia dirigida a establecer distinciones entre competidores. Esto es algo que funciona como motor de innovación, lo que produce una distinción en la producción similar a la distinción social en el acto de consumo (ver Veblen, 2007 [1899], Bourdieu, 1979; y Baudrillard, 1981).

Ante estas perspectivas adquieren valor las tesis menos lineales que apuntan a la existencia de participaciones múltiples y al carácter complejo del proceso de innovación (Freeman, 1987, 2004; von Hippel, 1988, 2006; Pinch \& Bijker, 1984; Latour et al., 2002a, 2002b), aunque se reserva cierto grado de acción (asimétrica) a la técnica. También se refuerzan las posturas que tienden a poner de relieve el papel de las profesiones y los especialistas (Flichy, 2003), al igual que el de los retóricos, en el desarrollo de funcionalidades. Además, el origen externo de algunas innovaciones muestra la importancia de la apertura de la empresa para promover la creación de nuevos productos. En suma, este análisis revela un modelo productivo dependiente de las coyunturas y de una interacción inter-empresarial que deja escaso margen para una efectiva participación espontánea a los consumidores.

\section{NOTAS}

* El autor quiere agradecer al Dr. Fernández-Esquinas su ayuda en la clasificación de términos y corrección de este texto.

1 Nos centraremos en el ámbito específicamente técnico y empresarial, de ahí que interacciones tecno-científicas con la Universidad, como por ejemplo las exploradas en la triple- hélice (Leydesdorff \& Etzkowitz, 1998), sean aquí eludidas, algo resultante de lo que podemos observar en la empresa.

2 Los estudios de gestión de innovación y de márketing como disciplina abordan tipos de intervención del marketing sobre la innovación y su relación con los ingenieros (ver Tidd et al., 2003; Nuchera et al., 2002; Griffin \& Hauser, 1996; y Keaveney,
Aceptado: 26 de junio de 2011 
2008). Estos estudios no suelen considerar un acercamiento externo al marketing y su relación al artefacto tecnológico. Este artículo se diferencia en la utilización de una perspectiva más flexible.

3 Principalmente Goffman (1981) para "marcos de referencia", y Star y Griesemer (1989) para "objetos fronterizos".

4 http://www.ndrive.com

5 http://www.infoportugal.pt

6 Tiene sesenta trabajadores y está presente en cuarenta países.

7 Una interacción con la Universidad, aunque no de carácter tecno-científico.

8 http://www.blomasa.com/

9 http://www.tomtom.com/

10 http://www.garmin.com

11 A veces hay procesos de contaminación entre los dos ámbitos, pero con dominio del funcional. Hay un caso inverso, pero sin relevancia técnica.

12 Hay una tendencia muy reciente para una mayor participación de usuarios líder en el desarrollo a través de foros on-line -ver "beta.ndrive.com"-, pero no tiene influencia sobre los productos en cuestión.

13 Hay casos raros de algunas funcionalidades propuestas por los consumidores en estos foros de internet, pero que finalmente no llegaron a adoptarse -ver "www.portalppc.com" y "www-gsmpt.net".

14 El caso del Iphone 4 (Apple) es un buen ejemplo: la antena del dispositivo aparece en una posición que se sobrepone por la mano que lo mantiene "naturalmente", provocando la pérdida de la señal. Ello se debió a un descuido en relación al código gestual estabilizado. El responsable de su desarrollo fue despedido.

15 Hermínio Martins (2006) utiliza el concepto de "profecía auto-realiza- da" como interpretación de la ley de Moore, la cual indica la duplicación de la capacidad de procesamiento de los microchips cada doce meses. Martins defiende que, en realidad, son las expectativas y los compromisos tácitos de la industria las que hacen efectiva esa pretendida ley.

16 En algunos aspectos, la posición más próxima del poder parece favorecer decisiones retóricas, aunque la importancia de las especializaciones y de los conocimientos no compartidos destacan una clara negociación con una simetría general basada en la confianza y legitimidad de los papeles desempeñados.

\section{BIBLIOGRAFÍA}

Aristóteles (1998): Retórica, Lisboa, Imprensa Nacional-Casa da Moeda.

Baudrillard, J. (1981[1972]): Para uma Crítica da Economia Política do Signo, Porto, Ed. 70.

Bourdieu, P. (1979): La distinction: critique sociale du jugement, Paris, Minuit.

Ellul, J. (1954): La technique ou l'enjeu du siècle, Paris, Armand Colin.

Fagerberg, J.; Mowery, D. C. y Nelson, R. R. (2005): The Oxford Handbook of Innovation, Oxford, Oxford University Press.

Flichy, P. (2003): L'Innovation Technique, Récents développements en sciences socials, vers une nouvelle théorie de l'innovation, Paris, Édition La Découvert.

Freeman, C. (1987): Technology Policy and Economic Performance: Lessons from Japan, Londres, Pinter.

Freeman, C. y Louçã, F. (2004): Ciclos e Crises no Capitalismo Global, das Revoluções Industriais à Revolução da Informação, Santa Maria da Feira, Edições Afrontamento.
Gell, A. (1998): Art and Agency, An Anthropological Theory, Oxford, Clarendon Press.

Gille, B. (1973): Histoire des Techniques, Paris, Gallimard.

Goffman, E. (1981): Les cadres de l'expérience, Paris, Édition de Minuit.

Griffin, A. y Hauser, J. R. (1996): "Integrating $R \& D$ and Marketing: a review and analysis of the literature", en Journal of Product Management, 13: 191-215.

Heilbroner, R. L. (1967): "Do Machines Make History?", Technology and Culture, Vol. 8, n. ${ }^{\circ}$ 3, pp. 335-345.

Horkheimer, M. y Adorno, T. W. (2002 [1944]: Dialectic of Enlightenment, Philosophical Fragments, Stanford, Stanford University Press.

Latour, B. (1992): "Where Are the Missing Masses? The Sociology of a Few Mundane Artifacts", en W. Bijker e J. Law (coord.), Shaping Technology/Building Society, Studies in Sociotechnical Chage, Massachusetts, The MIT Press, pp. 225-259.

Latour, B.; Akrich, M. y Callon, M. (2002a): "The Key to Success in Innovation, Part I, The Art of Interessement", International Journal of Innovation Management, Vol. 6, n. ${ }^{\circ} 2$, pp. 187-206.

Latour, B., Akrich, M. y Callon, M. (2002b): "The Key to Success in Innovation, Part I, The Art of Choosing Good Spokespersons", en International Journal of Innovation Management, Vol. 6, n. ${ }^{\circ}$, pp. 207-225.

Lendrevie, J.; Lindon, D.; Dionísio, P. y Rodrigues, J. V. (1996): Mercator, Teoria e Prática do Marketing, Lisboa, Publicações Dom Quixote.

Leydesdorff, L. y Etzkowitz, H. (1998): "The Triple Helix as a Model for Innovation Studies", Science and Public Policy, 25 (3), pp. 195-203.

Lien, M. E. (1997): Marketing and Modernity, Oxford, Berg. 
Keaveney, S. M. (2008): "The blame of the game: an attribution theory approach to market-engineer conflict in hightechnology company", Industrial Marketing Management, 37: 653-663.

Kline, S. J. y Rosenberg, N. (1986): "An overview of innovation", en Landau, R. y Rosenberg, N. (coord.), The positive sum strategy. Harnessing technology for economic growth, Washington, D.C.: National Academy Press, pp. 275-305.

Martins, H. y Garcia, J. L. (2006): "Introdução", Análise Social; Revista de Ciências Sociais da Universidade de Lisboa; Tecnologia; Perspectivas Críticas e Culturais, 181, Vol. XLI, $4 .^{\circ}$ semestre, pp. 941-956.

Martins, H. (2006): "Dilemas da República Tecnológica", Análise Social; Revista de Ciências Sociais da Universidade de Lisboa; Tecnologia; Perspectivas Críticas e Culturais, 181, Vol. XLI, $4 .^{\circ}$ semestre, pp. 959-979.
Mendonça, P. (2010): "Tecnologia e Retórica, para uma retórica material da função útil, o exemplo do telemóvel", Media \& Jornalismo, n. ${ }^{\circ}$ 16, Vol. 9, n. ${ }^{\circ}$, pp. 183-200.

Nuchera, A. H.; Morote, J. P. y Serrano, G. L. (2002): La Gestión de la Innovación y la Tecnología en las Organizaciones, Madrid, E. Pirâmide.

Pinch, T. y Bijker, W. (1984), "The Social Construction of Facts and Artifacts: Or How the Sociology of Science and the Sociology of Technology Might Benefit Each Other", Social Studies of Science, n. ${ }^{\circ} 14$, pp. 399-441.

Rodrigues, M. J.; Neves, A. y Godinho, M. M. (coord.) (2003): Para Uma Política de Inovação em Portugal, Lisboa, D. Quixote.

Schumpeter, J. A. (1996): Ensaios, Empresários, Inovação, Ciclos de Negócio e Evolução do Capitalismo, Oeiras, Celta Editores.
Star, S. y Griesemer, L. (1989): "Institutional Ecology, Translations and Boundary Objects: Amateurs and Professionals in Berkeley's Museum of Vertebrate Zoology (1907-1939)", Social Studies of Science, vol. 19, n. ${ }^{\circ}$ 3, pp. 387-420.

Stiegler, B. (1994): La Technique et Le Temps I, La faute d'Epiméthée, Paris, Galilée, Cité des Sciences et de I'Industrie.

Thévenot, L. (2006): L'Action au Pluriel, Sociologie des Régimes d'Engagement, Paris, Ed. La Découvert.

Tidd, J.; Bessant, J. y Pavitt, K. (2003): Gestão da Inovação, Integração das Mudanças Tecnológicas, do Mercado e Organizacionais, Lisboa, Monitor.

Veblen, T. (2007 [1899]): The Theory of the Leisure Class, Oxford, Oxford University Press.

Von Hippel, Eric (1988): The Sources of Innovation, Oxford, Oxford University Press.

Von Hippel, Eric (2006): Democratizing Innovation, Massachusetts, The MIT Press. 